\title{
Oxidative Coupling Copolycondensation of 2,6-Dimethylphenol with 2,5-Dimethylphenol: Highly Thermostable Poly(phenylene ether)
}

\author{
By Yuji ShIBASAKI, ${ }^{1, *}$ Kenta HoshI, ${ }^{1}$ Eiichi SUZUKI, ${ }^{1}$ Yutaka SHIRAISHI, ${ }^{2}$ \\ Yasumasa NORISUE, ${ }^{3}$ and Yoshiyuki OISHI ${ }^{1}$
}

2,5-Dimethylphenol (25DMP) was successfully copolymerized with 2,6-dimethylphenol (26DMP) using the di- $\mu$-hydroxo$\operatorname{bis}\left[\left(N, N, N^{\prime}, N^{\prime}\right.\right.$-tetramethylethylenediamine)copper (II)]chloride (CuCl-TMEDA) catalyst with 10 equiv (to copper ion) of tetramethylethylenediamine (TMEDA) in toluene at $20^{\circ} \mathrm{C}$ for $7 \mathrm{~h}$ under oxygen. Conversion of the two monomers was monitored by gas chromatography, which revealed that 25DMP was slightly less reactive than 26DMP. Infrared spectra of a series of the copolymers indicated that the composition of the copolymer could be controlled by the monomer feed ratio. The resulting copolymer shows a higher thermal air stability of about $70^{\circ} \mathrm{C}$ along with improved mechanical properties in comparison with the conventional poly(2,6-dimethyl-1,4-phenylene ether) (PPE). PPE with a methyl end group, prepared by the endcapping reaction of PPE with methyl iodide in the presence of potassium hydroxide in $N, N$-dimethylformamide, shows a higher thermal stability than the as-prepared PPE under air, and similar stability under nitrogen, indicating that thermal degradation mainly occurs from the polymer end group under air. Thus, the higher thermal stability of the copolymer is attributable to the 25DMP unit located at the end of the copolymer.

KEY WORDS: Oxidative Polymerization / 2,5-Dimethylphenol / 2,6-Dimethylphenol / Copper Catalyst / Degradation /

Poly(2,6-dimethyl-1,4-phenylene ether) (PPE) is a widely accepted engineering plastic used in a variety of fields because of its good mechanical, dimensional stabilities, low water uptake, and low dielectric characteristics. ${ }^{1}$ However, PPE is likely to decompose under exposure to ultraviolet radiation, resulting in yellowing. ${ }^{2}$ Yellowing is a fatal issue for organic polymer materials. Hindered amines are the typical antioxidant, which trap radical species to form stable nitroxy radical. ${ }^{3}$ Ultraviolet radiation stable PPE was also prepared by incorporating hindered amines as an additive. ${ }^{4}$ However, it is difficult to improve the ultraviolet radiation stability only by the chemical modification of PPE despite of the reports on highly thermostable crosslinked PPE resin. ${ }^{5}$ Furthermore, due to the low oxidative stability of the polymer, blending with another plastic such as polystyrene is required in order to decrease the glass transition temperature for injection processing. ${ }^{6}$ Thus, currently the potential of PPE remains unfulfilled in terms of application to industry.

Notably, none of the phenol derivatives, with the exception of 2,6-dimethylphenol, are available as conventional oxidative coupling polymerization monomers with a copper-amine catalyst because of radical propagation; thus development of a novel high performance PPE has been strictly limited to date.

Hay et al. reported that catalysis using $\mathrm{CuCl}$ with a 2-alkyl pyridine such as 2-tridecylpyridine was effective in the synthesis of a high-molecular-weight poly $(o$-cresol $) ;{ }^{7}$ however, branching could not be completely excluded. Higashimura et al. reported on the use of a tridentate copper catalyst, a $\mu-\eta^{2}: \eta^{2}$-peroxo dicopper (II) complex, in conjunction with a bulky 2,6-diphenylpyridine base for the regio-controlled polymerization of 2,5-dimethylphenol (25DMP), where the resulting polymer, poly(2,5-dimethyl-1,4-phenylene ether) (PPE25), had an average molecular weight $\left(M_{\mathrm{n}}\right)$ of 4,000 and a molecular weight distribution $\left(M_{\mathrm{w}} / M_{\mathrm{n}}\right)$ of $4.9 .^{8}$ Although this catalytic system could then be applied to the polymerization of other phenol derivatives such as $o$-cresol ${ }^{9} m$-cresol, ${ }^{10}$ and $p$ phenoxyphenol, ${ }^{11}$ perfect regio-regularity has not been realized. A mesoporous supported copper-amine catalyst was prepared and applied to the oxidative polymerization of 2,6dimethylphenol (26DMP) $)^{12}$ and $25 \mathrm{DMP},{ }^{13}$ in which a perfectly regio-controlled polymer was obtained. This catalyst was also applied to the regio-controlled oxidative polymerization of $o$ cresol, ${ }^{14}$ but the regio-regularity was only about $90 \%$. These results suggest that careful design of the catalytic environment is vital in achieving regio-selectivity in phenol polymerization. A more simple and homogeneous catalytic system based on $\mathrm{CuCl}$ with 150 equiv of 2-( $p$-tolyl)pyridine has also been employed for the polymerization, giving excellent regioregularity of the resulting PPE25; the melting temperature and the degree of crystallinity of the PPE25 were $310^{\circ} \mathrm{C}$ and $42 \%$, respectively. ${ }^{15}$ Very recently, a di- $\mu$-hydroxo$\operatorname{bis}\left[\left(N, N, N^{\prime}, N^{\prime}\right.\right.$-tetramethylethylenediamine)copper (II)]chlo-

\footnotetext{
${ }^{1}$ Department of Chemistry \& Bioengineering, Iwate University, 4-3-5 Ueda, Morioka 020-8551, Japan

${ }^{2}$ Mitsubishi Engineering-Plastics Corporation, 5-6-2, Higashiyawata, Hiratsuka 254-0016, Japan

${ }^{3}$ Mitsubishi Gas Chemical Co., Inc. Specialty Chemicals Company Planning \& Development Division, Mitsubishi Building 5-2, Marunouchi 2-Chome, Chiyoda-ku, Tokyo 100-8324, Japan

*To whom correspondence should be addressed (Tel: +81-19-621-6322, Fax: +81-621-6322, E-mail: yshibasa@iwate-u.ac.jp).
} 
ride ( $\mathrm{CuCl}$-TMEDA) catalyst was found to promote the regiocontrolled oxidative polymerization of 25DMP with less base additive required (tetramethylethylenediamine 3 equiv to $\mathrm{CuCl}$ ), although the regio-regularity was not perfect. ${ }^{16}$ PPE25 is a semi-crystalline polymer that shows improved thermal and mechanical properties compared with the conventional PPE, but suffers from poor solubility in typical organic solvents; thus, the molecular weight of PPE25 is always about 5,000 or below. We anticipated that copolymerization of 25DMP with 26DMP would provide better thermal and mechanical properties than those of conventional PPE. By such a method, the potential of PPE could be realized without the need for blending with another thermoplastic.

Herein, we report on the oxidative coupling copolymerization of 25DMP with 26DMP with a CuCl-TMEDA catalyst and the properties of the resulting copolymer.

\section{EXPERIMENTAL}

\section{Reagents}

25DMP and 26DMP were purified by recrystallization with hexane, followed by sublimation, and stored under nitrogen. The CuCl-TMEDA complex was purchased from Tokyo Chemical Industry Co., Ltd. and used as received. All the other reagents and solvents were used as received.

\section{Polymerization}

Typical procedure of copolymerization with PPE2 $5_{\mathrm{x}}$ (x: molar ratio of 25DMP): Into a two-necked flask were added 26DMP (9.80 g, 80.2 mmol), 25DMP (0.20 g, $1.63 \mathrm{mmol})$, and toluene $(130 \mathrm{~mL}) . \mathrm{CuCl}(\mathrm{OH}) \mathrm{TMEDA} \quad(0.952 \mathrm{~g}, 2.05 \mathrm{mmol}$, $2.5 \mathrm{~mol} \%$ to the monomer) and TMEDA (4.75 g, $40.9 \mathrm{mmol})$ were added in this order. The flask was purged with oxygen two times and the mixture was stirred at $20^{\circ} \mathrm{C}$ for $7 \mathrm{~h}$. The resulting solution was poured into methanol $(1.8 \mathrm{~L})$ containing $20 \mathrm{~mL}$ of concentrated aqueous hydrogen chloride to precipitate the polymer. The precipitate was collected, dissolved in toluene $(170 \mathrm{~mL})$, and reprecipitated with methanol $(1.2 \mathrm{~L})$ containing $10 \mathrm{~mL}$ of concentrated aqueous hydrogen chloride. This reprecipitation procedure was repeated two more times to ensure removal of copper ions. The polymer was collected and dried at $100{ }^{\circ} \mathrm{C}$ for $12 \mathrm{~h}$ under vacuum (PPE25 $2.21 \mathrm{~g}, 85.4 \%$ yield).

\section{Kinetic Study}

In the next stage and in the following order, 26DMP $(1.00 \mathrm{~g}$, $8.26 \mathrm{mmol}), 25 \mathrm{DMP}(1.00 \mathrm{~g}, 8.26 \mathrm{mmol})$, octadecane $(0.210 \mathrm{~g}$, $0.826 \mathrm{mmol})$ and toluene $(25 \mathrm{~mL}), \mathrm{CuCl}(\mathrm{OH})$ TMEDA $(0.192 \mathrm{~g}$, $0.413 \mathrm{mmol}, 2.5 \mathrm{~mol} \%$ to the monomer) and TMEDA $(0.959 \mathrm{~g}$, $8.26 \mathrm{mmol}$ ) were added to a two-necked flask. The flask was purged with oxygen two times, and the polymerization solution was fractionated in the set time to measure the consumption of the monomers by GC using octadecane as an internal standard.

\section{End Functionalization of PPE}

Into a Erlenmeyer flask PPE $\left(0.122 \mathrm{~g}, M_{\mathrm{n}}=1,300\right), \mathrm{KOH}$
$(0.65 \mathrm{~g}, 11 \mathrm{mmol})$, toluene $(6 \mathrm{~mL})$, and $N, N$-dimethylformamide $(6 \mathrm{~mL})$ were added at $0{ }^{\circ} \mathrm{C}$, and the mixture was stirred until the polymer was completely dissolved. Methyl iodide $(1.40 \mathrm{~g}, 10.0 \mathrm{mmol})$ was then added to the solution, and reacted at $0{ }^{\circ} \mathrm{C}$ for $2 \mathrm{~h}$. The reaction solution was transferred to a separating funnel containing water, and the organic compounds were extracted with dichloromethane twice. The organic layer was concentrated by a rotary evaporator, and dropped into methanol to precipitate the polymer. The resulting precipitate was dried at $150{ }^{\circ} \mathrm{C}$ for $12 \mathrm{~h}$ under reduced pressure $(0.048 \mathrm{~g}$, $40 \%$ yield, almost $100 \%$ functionality). The end functionality was determined by the complete disappearance of the hydroxyl polymer end group at $4.238 \mathrm{ppm}$ in the ${ }^{1} \mathrm{H}$ NMR spectrum and the corresponding appearance of the methyl ether proton at $3.669 \mathrm{ppm}$.

\section{Estimation of Polymer Composition}

The composition of the copolymers was determined from the intensity ratio of $\mathrm{C}-\mathrm{H}$ wagging absorption of two segments, 26DMP and 25DMP, in the IR spectrum. Calibration was performed using six polymer samples prepared by blending PPE and PPE25 homopolymers.

\section{Film Preparation}

On a press-molding machine, SUS, Teflon $(0.1 \mathrm{~mm}$ in thickness), Upilex (1 mm in thickness), and an aluminum mold $(10(\mathrm{~L}) \times 110(\mathrm{~W}) \times 2(\mathrm{H}) \mathrm{mm})$ were placed in this order. The mold was filled with the well-crushed polymer powder, and the film thickness was adjusted with Teflon rod that was placed upon the sample. Upilex, Teflon, and SUS plates were placed on it, and the sample was pressed at $5 \mathrm{MPa}$ at $220^{\circ} \mathrm{C}$ for $10 \mathrm{~min}$, followed by at $280^{\circ} \mathrm{C}$ for $20 \mathrm{~min}$ at this pressure.

\section{Measurements}

Fourier transform infrared (IR) spectra were measured with a Jasco IR-5500 (Jasco Co., Ltd.) by transmittance absorption spectroscopy ( $\mathrm{KBr}$ tablet method). Number and weight average molecular weights $\left(M_{\mathrm{n}}\right.$ and $\left.M_{\mathrm{w}}\right)$ were measured by gel permeation chromatography (GPC) on a Tosoh HLC-8120 GPC equipped with consecutive polystyrene gel columns (TSK-GEL $\mathrm{GMH}_{\mathrm{HR}}-\mathrm{M}$ and $\mathrm{GMH}_{\mathrm{HR}}-\mathrm{N}$ ) at $40^{\circ} \mathrm{C}$ eluted with chloroform at a flow rate of $1.0 \mathrm{~mL} \mathrm{~min}^{-1}$ calibrated by standard polystyrene samples. Gas chromatography (GC) was performed on a Shimadzu GC-2010 equipped with a packed silica gel column (Chromosorb W 60/80 AW) at $180{ }^{\circ} \mathrm{C}$ with a helium carrier using a hydrogen-flame ionization detector. Octadecane (GC standard grade) was used as an internal reference. Nuclear magnetic resonance (NMR) was performed on a Bruker AC-400P spectrometer at $400 \mathrm{MHz}$ for ${ }^{1} \mathrm{H}$ and $100 \mathrm{MHz}$ for ${ }^{13} \mathrm{C}$ measurement. Deuterated chloroform $\left(\mathrm{CDCl}_{3}\right)$ was used as a solvent with tetramethylsilane as an internal reference. Thermal analyses were performed on a Seiko thermal analyzer (SCC 5200 system) at a heating rate of $10^{\circ} \mathrm{C} / \mathrm{min}$ for thermogravimetric analysis (TGA by TG/DTA 320) and $2^{\circ} \mathrm{C} / \mathrm{min}$ for dynamic mechanical analysis (DMA by DMS 210) under air or nitrogen. Differential scanning 


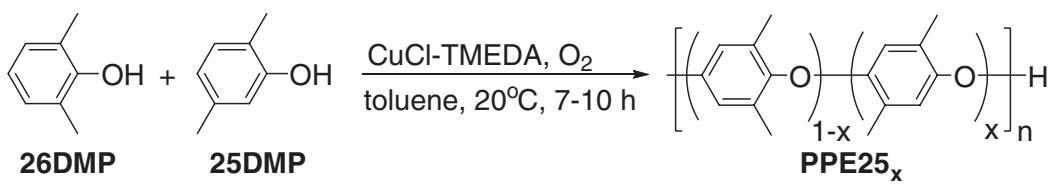

Scheme 1.

Table I. Copolymerization of 26DMP with 25DMPa

\begin{tabular}{|c|c|c|c|c|c|c|c|c|}
\hline Sample & Monomer & Polymer & $\begin{array}{l}\text { Feed } \\
/ \mathrm{mol} \%\end{array}$ & $\begin{array}{l}\text { Time } \\
/ \mathrm{h}\end{array}$ & $\begin{array}{c}\text { Yield } \\
/ \%\end{array}$ & $\begin{array}{l}\text { Composition }^{\mathrm{b}} \\
\text { 26DMP: } \\
\text { 25DMP }\end{array}$ & $M_{\mathrm{n}}{ }^{\mathrm{c}}$ & $M_{\mathrm{w}} / M_{\mathrm{n}}{ }^{\mathrm{c}}$ \\
\hline 1 & 25DMP & PPE25 & 100 & 8 & 55 & $0: 100$ & - & - \\
\hline 2 & 26DMP+25DMP & PPE25 70 & $30: 70$ & 8 & 52 & $54: 44$ & 8,500 & 3.4 \\
\hline 3 & 26DMP+25DMP & PPE25 50 & $50: 50$ & 8 & 56 & $63: 37$ & 7,400 & 3.1 \\
\hline 4 & 26DMP+25DMP & PPE25 30 & $70: 30$ & 10 & 73 & $62: 38$ & 7,400 & 3.0 \\
\hline 5 & 26DMP+25DMP & PPE25 20 & $80: 20$ & 7 & 61 & $86: 14$ & 7,800 & 2.3 \\
\hline 6 & 26DMP+25DMP & PPE25 10 & $90: 10$ & 7 & 71 & $92: 8$ & 7,200 & 2.2 \\
\hline 7 & 26DMP+25DMP & PPE25 5 & $95: 5$ & 7 & 86 & $93: 7$ & 6,900 & 2.0 \\
\hline 8 & 26DMP+25DMP & $\mathrm{PPE}_{2} 5_{3}$ & $97: 3$ & 7 & 67 & $94: 6$ & 10,000 & 2.0 \\
\hline 9 & 26DMP+25DMP & PPE25 2 & $98: 2$ & 7 & 86 & $95: 5$ & 10,400 & 2.0 \\
\hline 10 & 26DMP+25DMP & PPE25 1 & $99: 1$ & 7 & 71 & $97: 3$ & 8,500 & 2.0 \\
\hline 11 & 26DMP & PPE & 100 & 1.5 & 67 & $100: 0$ & 10,300 & 2.2 \\
\hline
\end{tabular}

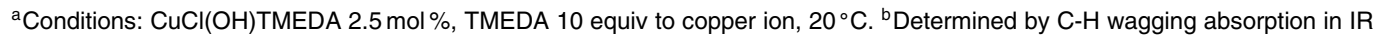
spectrum. ${ }^{\mathrm{C}}$ Determined by GPC $\left(\mathrm{CHCl}_{3}\right.$, PSt standard).

calorimetry (DSC) was analyzed on Shimadzu DSC-60 at a heating rate of $20^{\circ} \mathrm{C} / \mathrm{min}$ under nitrogen. The refractive index of the polymer films was measured on an Atago DR-M4 Abbe refractometer. Press-molding of the polymer samples was performed on a Toyoseiki Mini Test Press-10 at $5 \mathrm{MPa}$ pressure and $280^{\circ} \mathrm{C}$ for $20 \mathrm{~min}$. The mechanical properties of the polymer films $(50 \mathrm{~mm} \times 5 \mathrm{~mm})$ were measured by a Shimadzu AGS-D mechanical analyzer at a pulling rate of $10 \mathrm{~mm} / \mathrm{min}$. Measurement of the dielectric constant $(\varepsilon)$ and dielectric loss $(\tan \delta)$ was performed by a cavity resonance method.

\section{RESULTS AND DISCUSSION}

\section{Copolymerization}

We selected the CuCl-TMEDA catalyst because this catalyst is effective in the regio-controlled oxidative coupling polymerization of both 25DMP and 26DMP (Scheme 1). Table I summarizes the results of the polymerization. As already reported, a regio-regular homopolymer is available from 25DMP in moderate yield as a white powder (sample 1), ${ }^{16}$ which precipitates during polymerization. For molding by a press machine, the polymer samples must be effectively crushed into a powder to remove any air voids and to decrease the melt viscosity; a $M_{\mathrm{n}}$ value of around 10,000 is favorable. Thus, various copolymers (PPE25 $5_{\mathrm{x}}$, where $\mathrm{x}$ indicates the mol \% of 25DMP in the feed) with moderate $M_{\mathrm{n}}$ (around $10,000)$ were prepared by varying the polymerization time. When the feed of 25DMP was increased, the yield of the copolymer increased. The composition of the copolymer was estimated by the wagging absorption of the aromatic C-H bonds apparent at 857 and $885 \mathrm{~cm}^{-1}$ for PPE and PPE25, respectively. The composition of the copolymer was controlled

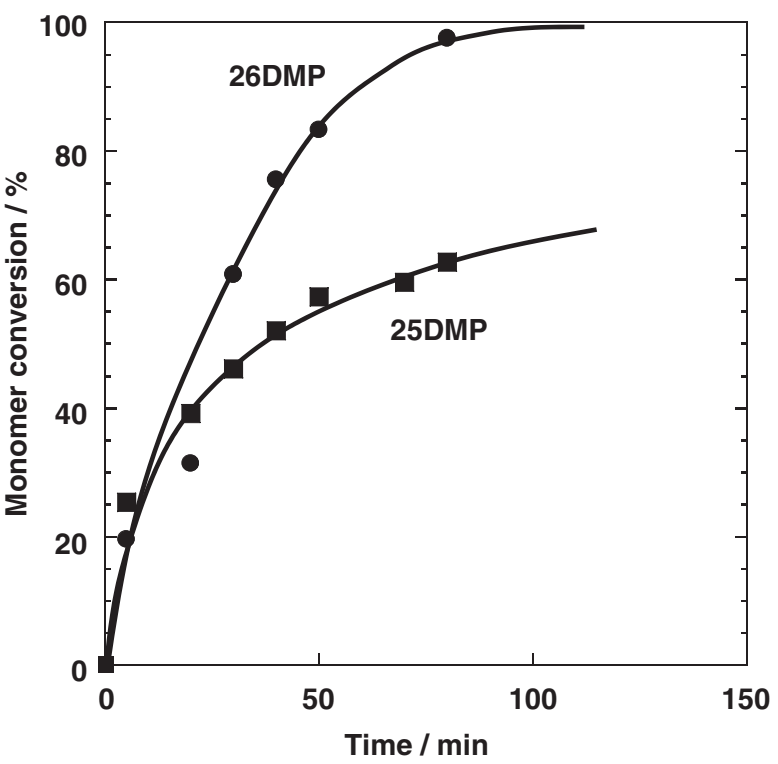

Figure 1. Time-conversion curves of 26DMP and 25DMP in the copolymerization reaction (1:1 in molar ratio).

by the monomer feed ratio, but the ratio of 25DMP in the copolymer gradually decreased in the high feed region (sample 2 and 3$)$.

In order to investigate the copolymerization behavior in detail, the monomer conversions were monitored by GC using octadecane as an internal standard. As shown in Figure 1, both monomers were similarly converted into the polymer chain in periods of time up to $30 \mathrm{~min}$, but after that time, 26DMP was favorably polymerized. These results indicated that the rate of the oxidation of 25DMP is slower than that of 26DMP. In other 


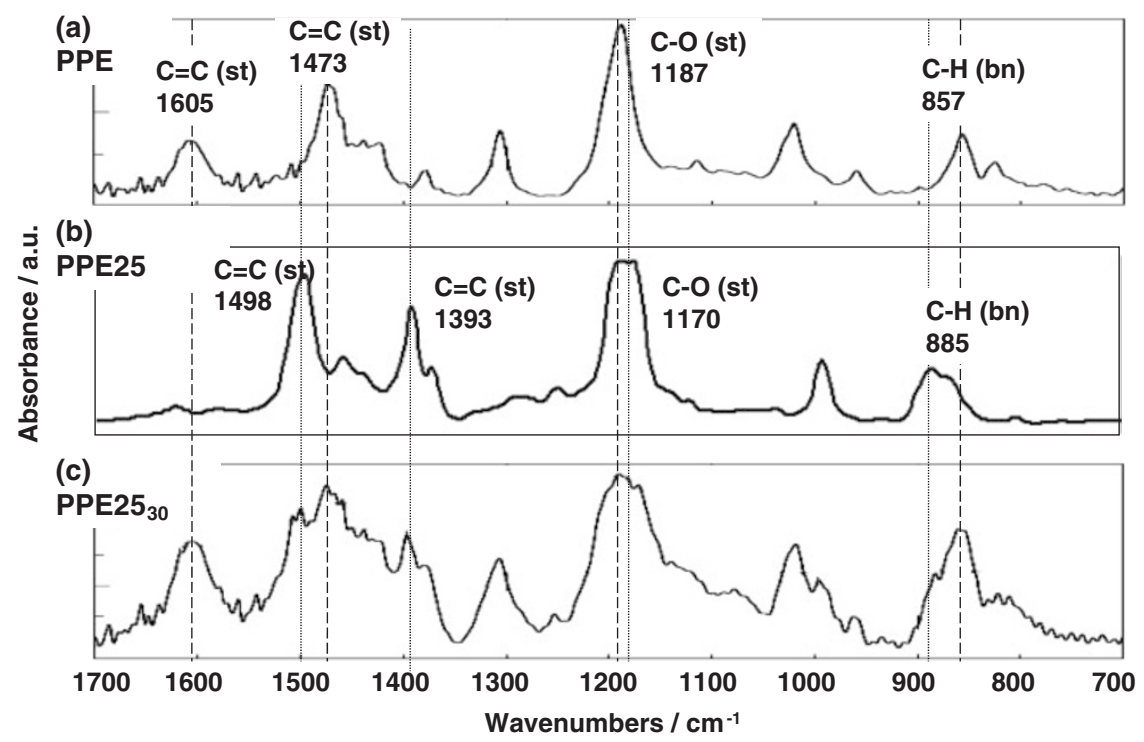

Figure 2. IR spectra of (a) sample 11 (PPE), (b) sample 1 (PPE25), and (c) sample 4 (PPE25 ${ }_{30}$ ). The dashed and broken lines indicate the absorption from PPE and PPE25, respectively.

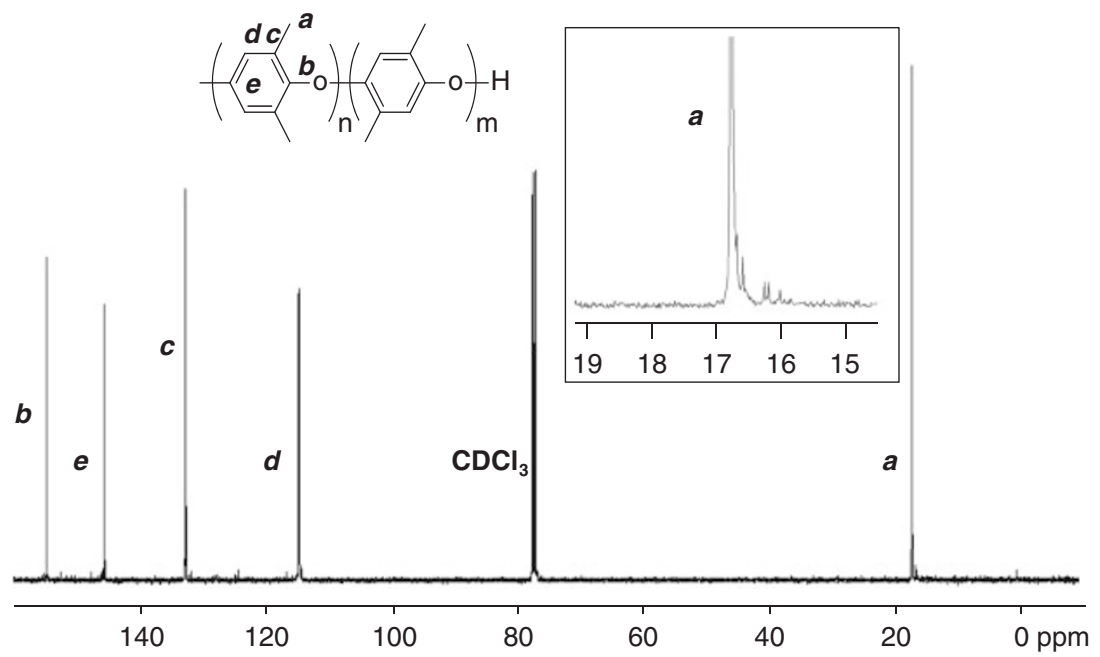

Figure 3. ${ }^{13} \mathrm{C}$ NMR spectrum and expanded region (inset, $14-19 \mathrm{ppm}$ ) of sample 8 (PPE253).

words, 25DMP is more stable than 26DMP under the present oxidation conditions.

\section{Characterization of the Copolymer}

The structure of the copolymer was characterized by IR and NMR analysis. Figure 2 depicts the IR spectra of (a) PPE, (b) PPE25, and (c) PPE25 30 . In the IR spectrum of the conventional PPE, absorption bands can be observed at 1473, 1187, $857 \mathrm{~cm}^{-1}$, assignable to $\mathrm{C}=\mathrm{C}$ stretching, $\mathrm{C}-\mathrm{O}-\mathrm{C}$ stretching, and $\mathrm{C}-\mathrm{H}$ bending, respectively. In contrast, these bands were observed for PPE25 at 1498, 1170, and $885 \mathrm{~cm}^{-1}$, respectively. The copolymer PPE25 30 has absorption bands corresponding to both PPE and PPE25 units, from which the composition of the two monomers in the copolymer was estimated to be about 62:38 (as calculated from $\mathrm{C}-\mathrm{H}$ bending modes). Thus, the composition of the copolymer can be controlled by the monomer feed ratio in copolymerization.

Because PPE25 $x$ with a high molar ratio of 25DMP do not have sufficient solubility in $\mathrm{CDCl}_{3}$, a PPE25 sample of lower 25DMP content was selected for analysis by ${ }^{13} \mathrm{C} \mathrm{NMR}$ spectroscopy. Figure 3 depicts the spectrum of $\mathrm{PPE} 25_{3}$ and its expanded region (14 to $19 \mathrm{ppm}$ ) in $\mathrm{CDCl}_{3}$ at $20^{\circ} \mathrm{C}$. Strong sets of signals are observed at 16.76, 114.2, 132.5, 145.4, $154.7 \mathrm{ppm}$, which are assignable to the 26DMP unit in the copolymer. In the expanded spectrum, smaller signals are observed at $16.67,16.59,16.24,16.19$, and $15.99 \mathrm{ppm}$. Since the ${ }^{13} \mathrm{C}$ NMR spectrum of PPE25 shows methyl carbon signals at $16.2 \mathrm{ppm}$, the observation of these small signals indicates a plural sequence of 26DMP and 25DMP in the copolymer as shown in Figure 4. 

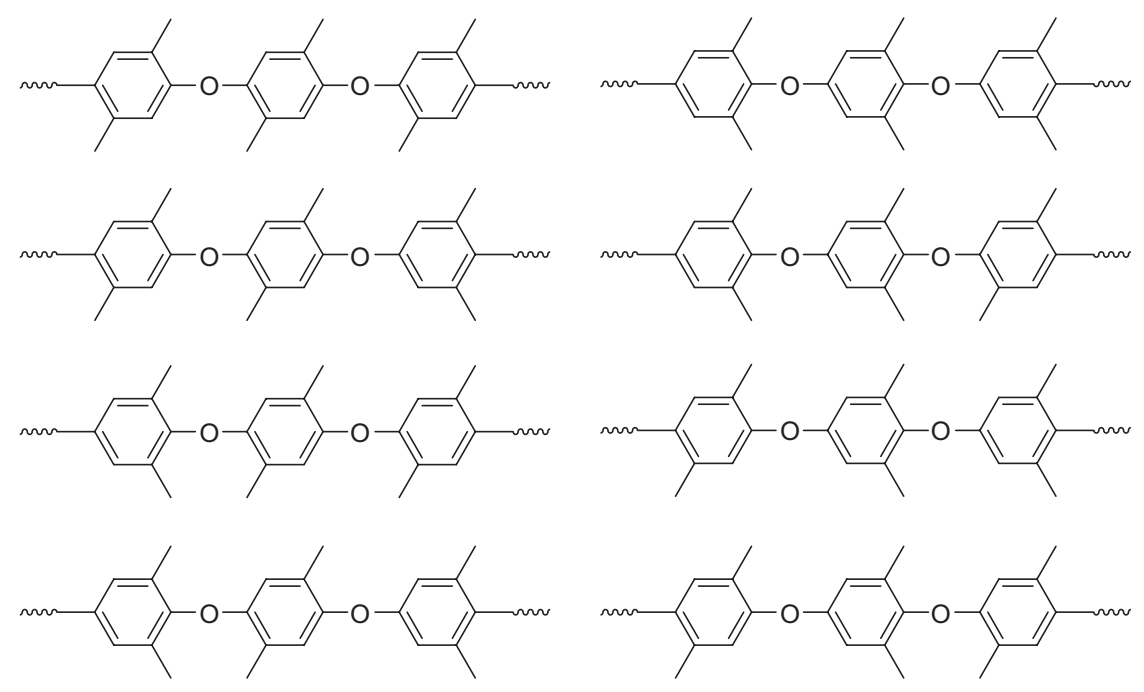

Figure 4. Possible sequences of the copolymer.

Table II. Thermal properties of polymers PPE25 ${ }^{a}$

\begin{tabular}{ccccccc}
\hline Sample & Abbreviation & $\begin{array}{c}T_{\mathrm{g}}^{\mathrm{b}} \\
/{ }^{\circ} \mathrm{C}\end{array}$ & $\begin{array}{c}T_{\mathrm{d} 5} / \mathrm{Air}^{\mathrm{c}} \\
/{ }^{\circ} \mathrm{C}\end{array}$ & $\begin{array}{c}T_{\mathrm{d} 10} / \mathrm{Air}^{\mathrm{c}} \\
/{ }^{\circ} \mathrm{C}\end{array}$ & $\begin{array}{c}T_{\mathrm{d} 5} / \mathrm{N}_{2}{ }^{\mathrm{c}} \\
/{ }^{\circ} \mathrm{C}\end{array}$ & $\begin{array}{c}T_{\mathrm{d} 10} / \mathrm{N}_{2}{ }^{\mathrm{c}} \\
/{ }^{\circ} \mathrm{C}\end{array}$ \\
\hline 2 & PPE25 $_{70}$ & 183 & 402 & 426 & 406 & 421 \\
3 & PPE25 $_{50}$ & 190 & 438 & 446 & 432 & 442 \\
4 & PPE25 $_{30}$ & 197 & 437 & 446 & 439 & 446 \\
5 & PPE25 $_{20}$ & 197 & 448 & 455 & 443 & 450 \\
6 & PPE25 $_{10}$ & 204 & 446 & 451 & 442 & 446 \\
7 & PPE25 $_{5}$ & 200 & 452 & 457 & 446 & 450 \\
8 & PPE25 $_{3}$ & 205 & 449 & 452 & 444 & 448 \\
9 & PPE25 $_{2}$ & 207 & 448 & 454 & 446 & 450 \\
10 & PPE25 & 207 & 448 & 454 & 444 & 445 \\
11 & PPE & 210 & 380 & 401 & 430 & 441 \\
\hline
\end{tabular}

${ }^{a}$ PPEx indicates the feed of 25DMP in $x .{ }^{b}$ Determined by DSC $\left(\mathrm{N}_{2}\right.$, $\left.20^{\circ} \mathrm{C} / \mathrm{min}\right)$. ${ }^{\mathrm{C}}$ Determined by TGA $\left(\mathrm{N}_{2}, 10^{\circ} \mathrm{C} / \mathrm{min}\right)$.

\section{Thermal Stability}

The thermal stability of the copolymer was investigated by TGA. Table II summarizes the results of the thermostability of the homo- and copolymers. It is noticeable that the inclusion of only $1 \mathrm{~mol} \%$ of $25 \mathrm{DMP}$ significantly increased the thermostability to about $70^{\circ} \mathrm{C}$ under air. Because both of the high reproducibility of this experimental result and the same isolation process of PPE and PPE25, the higher thermostability of PPE25 1 can be attributable to its nature, not the impurity. When the feed molar ratio of 25DMP was increased to $5 \mathrm{~mol} \%$, the thermostability of the copolymer attained a maximum value of $452{ }^{\circ} \mathrm{C}$. Figure 5 shows the $5 \mathrm{wt} \%$-loss temperature $\left(T_{\mathrm{d} 5}\right)$ under air and the glass transition temperature of the copolymer plotted against the monomer feed ratio. The thermal stability of the copolymer sharply increases with an initial increase in the feed of 25DMP, and then gradually drops to $438^{\circ} \mathrm{C}$ with further increases. As we already reported in literature, CuCl-TMEDA catalyst does not afford perfectly regio-controlled PPE25 homopolymer, ${ }^{15}$ the drop of thermostability in higher feed of 25DMP could be arisen by the unfavorable radical coupling site; $\mathrm{C}-\mathrm{C}$ or $\mathrm{C}-\mathrm{O}$ coupling at ortho

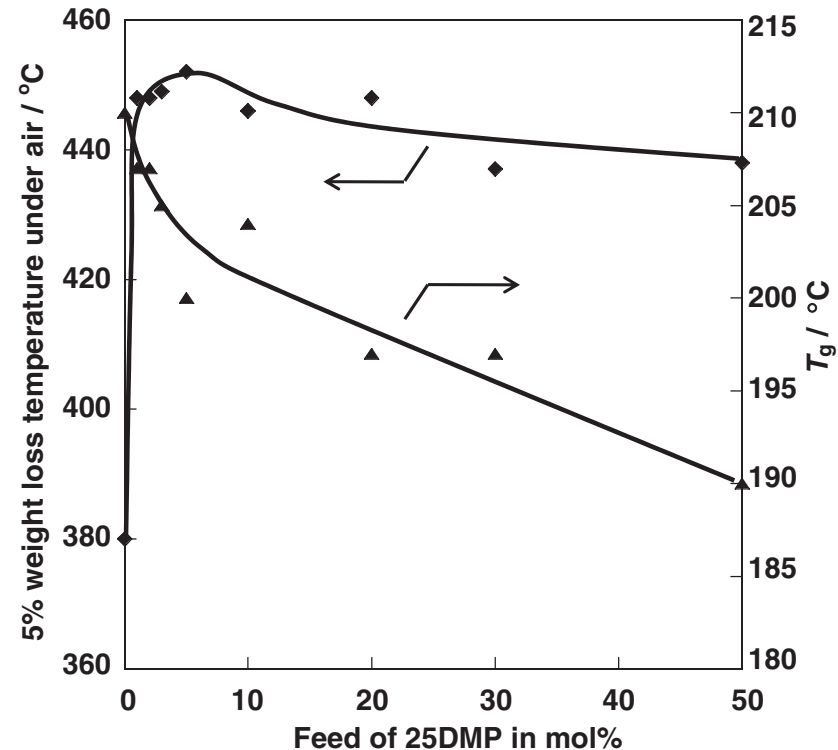

Figure 5. $T_{\mathrm{d} 5}$ and $T_{\mathrm{g}}$ of copolymers plotted against the feed composition of 25DMP.

position of $\mathrm{OH}$ moiety. In contrast, the glass transition temperature gradually decreases with increasing 25DMP, finally dropping to $190{ }^{\circ} \mathrm{C}$ for a $50 \mathrm{~mol} \%$ feed of $25 \mathrm{DMP}$, probably due to the shorter repeating number of the 26DMP unit in the copolymer. Thus, the copolymer could be processed at a lower temperature without the addition of another thermoplastic, which is generally required for conventional PPE in order to decrease its glass transition temperature sufficiently below the point of thermal decomposition.

TGA profiles of the conventional PPE (sample 11) and the copolymer PPE25 3 (sample 8) under air are shown in Figure 6. The value of $T_{\mathrm{d} 5}$ of $\mathrm{PPE}$, is $380^{\circ} \mathrm{C}$, which is $69^{\circ} \mathrm{C}$ lower than that of PPE2 $5_{3}$. It is noticeable that the conventional PPE gains its weight at $278^{\circ} \mathrm{C}$ by oxidation and reduces its weight from $358^{\circ} \mathrm{C}$, although no weight gain was observed for PPE $25_{3}$. 


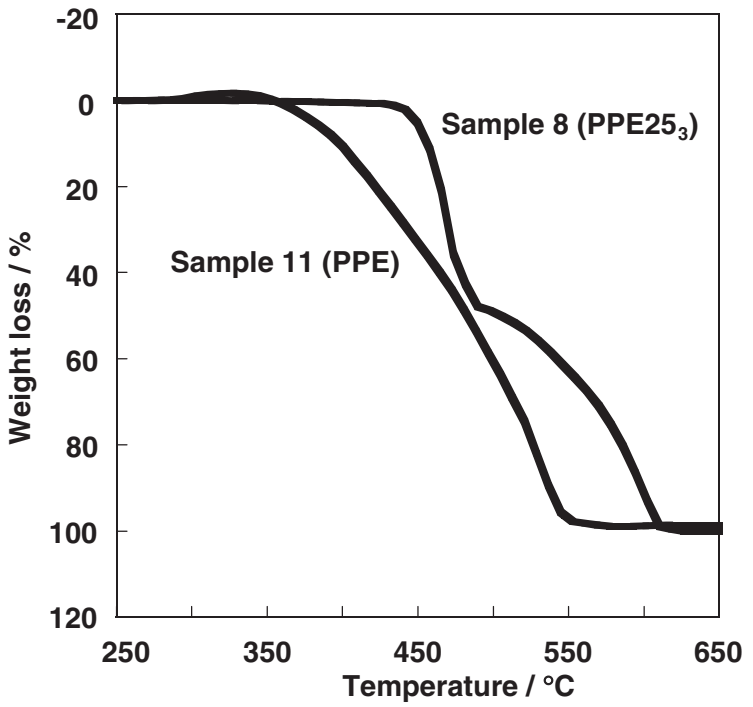

Figure 6. TGA profiles of (a) sample 11 (PPE) and (b) sample 8 (PPE25 $)$ $\left(10^{\circ} \mathrm{C} / \mathrm{min}\right.$ under air).

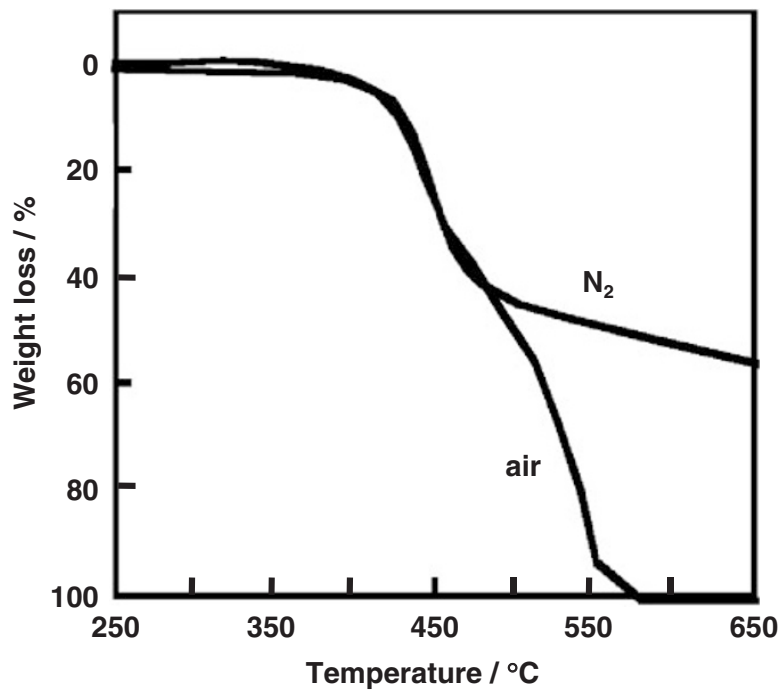

Figure 7. TGA profiles of PPE end functionalized with methyl iodide under air and nitrogen at a heating rate of $10^{\circ} \mathrm{C} / \mathrm{min}$.

Similar weight gain of PPE under air was also reported by A. Davis. ${ }^{17}$ The present results clearly indicate that copolymer has higher oxidation stability than PPE under air.

In order to determine the degradation mechanism of the copolymer, a fully end-functionalized PPE was prepared with methyl iodide. The value of $T_{\mathrm{d} 5}$ of the as-prepared PPE $(\mathrm{OH}$ function at the terminal) was 380 and $430{ }^{\circ} \mathrm{C}$ under air and nitrogen, respectively. In contrast, the TGA profiles of the endfunctionalized PPE $\left(\mathrm{OCH}_{3}\right.$ function) under air and nitrogen are comparable and the $T_{\mathrm{d} 5}$ was about $430{ }^{\circ} \mathrm{C}$ in both cases. Pickett reported that the oxidation rate of the end capped PPE was lower than that of uncapped PPE. ${ }^{18}$ Based on these results and information from the literature, thermal decomposition of the PPE mainly could occur from the polymer end group under air and from the main chain under nitrogen (Figure 7).
Table III. Calculated total energy ${ }^{\mathrm{a}}$

\begin{tabular}{cllc}
\hline Run & Substrates $^{\mathrm{b}}$ & \multicolumn{1}{c}{$\begin{array}{c}E_{0}^{\mathrm{c}} \\
/ \text { Hartree }\end{array}$} & $\begin{array}{c}\Delta E^{\mathrm{d}} \\
/ \mathrm{KJ} \mathrm{mol}^{-1}\end{array}$ \\
\hline 1 & $\mathrm{H}$ & -0.502257 & \\
2 & $26 \mathrm{DMP}$ & -386.2141 & 322.599 \\
3 & $26 \mathrm{DMP} *$ & -385.5759 & \\
4 & 26DMP-D & -771.2091 & 303.652 \\
5 & 26DMP-D* & -770.5789 & \\
6 & 25DMP & -386.2144 & 329.927 \\
7 & 25DMP* & -385.5734 & \\
8 & 25DMP-D & -771.2093 & 310.232 \\
9 & 25DMP-D* & -770.5764 & \\
\hline
\end{tabular}

${ }^{a}$ Calculated by DFT method (B3LYP/6-311++G(d,p)). ${ }^{\mathrm{b}}$ The abbreviation is as follows: H: hydrogen, 26DMP: 2,6-dimethylphenol, 26DMP*: 2,6dimethylphenoxy radical, 26DMP-D: 2,6-dimethylphenol dimer, 26DMP-D*: 2,6-dimethylphenol dimer radical, 25DMP: 2,5-dimethylphenol, 25DMP*: 2,5-dimethylphenoxy radical, 25DMP-D: 2,5-dimethylphenol dimer, 25DMP-D*: 2,5-dimethylphenol dimer radical. " Calculated total energy.

${ }^{\mathrm{d}}$ Dissociation energy of phenol derivatives into the radical species.

These results suggested that the high thermostability of PPE25 $x$, especially under air, is a result of the polymer end group, which could not be the 26DMP unit; thus, 25DMP mainly comprises the end units.

The higher stability of the copolymer with a 25DMP unit at the polymer chain end can be explained based on the dissociation energy, $\Delta E$, of the $\mathrm{OH}$ group. To this end, the dissociation energy was estimated by a density functional theory (DFT) calculation (B3LYP/6-311++G(d, p) basis set). Table III summarizes the dissociation energy of the $\mathrm{OH}$ group in the monomeric and dimeric forms of 26DMP and 25DMP. In both cases, the dissociation energy, $\Delta E$, of the 25DMP derivative is about $7 \mathrm{~kJ} / \mathrm{mol}$ higher than that of the 26DMP derivative, implying a higher oxidation stability for the 25DMP unit. This estimation agrees well with the results of timeconversion curves by GC, in which 25DMP is more stable under oxidation conditions. One possible explanation of the higher thermal stability of the copolymers in comparison to that of PPE can be offered by the structure of the polymer end. If thermal degradation occurs from the polymer chain end, having the more thermostable 25DMP unit at the polymer end group should contribute to greater thermal stability overall. Therefore, the probability of having a 25DMP unit at the polymer chain end is much higher than that of 26DMP.

\section{Mechanical Properties}

Table IV summarizes the mechanical properties of the copolymer. Conventional PPE shows the tensile strength, tensile modulus, and the elongation at break to be $64 \mathrm{MPa}$, $2.9 \mathrm{GPa}$, and $3.8 \%$, respectively. These values are improved to $80 \mathrm{MPa}, 4.0 \mathrm{GPa}$, and $3.6 \%$ by copolymerization with $2 \mathrm{~mol} \%$ of 25DMP. A further increase in the feed of 25DMP resulted in a decrease in the mechanical strength and the formation of a brittle film, probably as a consequence of the rigid-rod structure of the PPE25 segment.

Digital camera images of the tested sample films are shown in Figure 8. Whereas conventional PPE was partially carbonized under the molding conditions $\left(280^{\circ} \mathrm{C}, 5 \mathrm{MPa}, 20 \mathrm{~min}\right)$, 
Table IV. Mechanical properties of polymer ${ }^{\mathrm{a}}$

\begin{tabular}{rcccc}
\hline Sample & Abbreviation & $\begin{array}{c}\text { Strength }^{\mathrm{b}} \\
/ \mathrm{MPa}\end{array}$ & $\begin{array}{c}\text { Modulus }^{\mathrm{c}} \\
/ \mathrm{GPa}\end{array}$ & $\begin{array}{c}\text { Elongation }^{\mathrm{d}} \\
/ \%\end{array}$ \\
\hline 7 & PPE25 $_{5}$ & 67 & 3.3 & 3.4 \\
8 & $\mathrm{PPE}_{3}$ & 61 & 4.0 & 2.2 \\
9 & $\mathrm{PPE}_{3}$ & 80 & 4.0 & 3.6 \\
10 & $\mathrm{PPE}_{2}$ & 74 & 3.9 & 2.6 \\
11 & $\mathrm{PPE}$ & 64 & 2.9 & 3.8 \\
\hline
\end{tabular}

${ }^{a}$ All the films were molded at $5 \mathrm{MPa}$ at $280^{\circ} \mathrm{C}$ for $20 \mathrm{~min}$. ${ }^{\mathrm{b}}$ Tensile strength. ${ }^{\mathrm{c} T e n s i l e ~ m o d u l u s .}{ }^{\mathrm{d}}$ Elongation at break.

(a) PPE

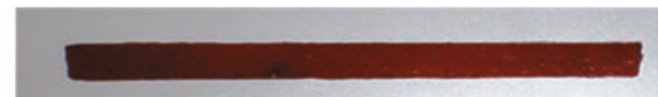

(b) PPE25

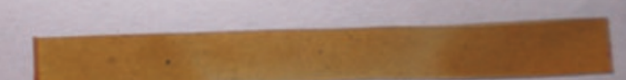

(c) $\mathrm{PPE}_{25}$

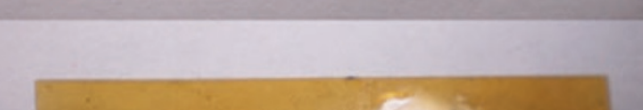

(d) PPE25

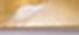
(PPE), (b) sample 10 (PPE251), (c) sample 9 (PPE25 $)$, and (d) sample 8 (PPE25 3 ).

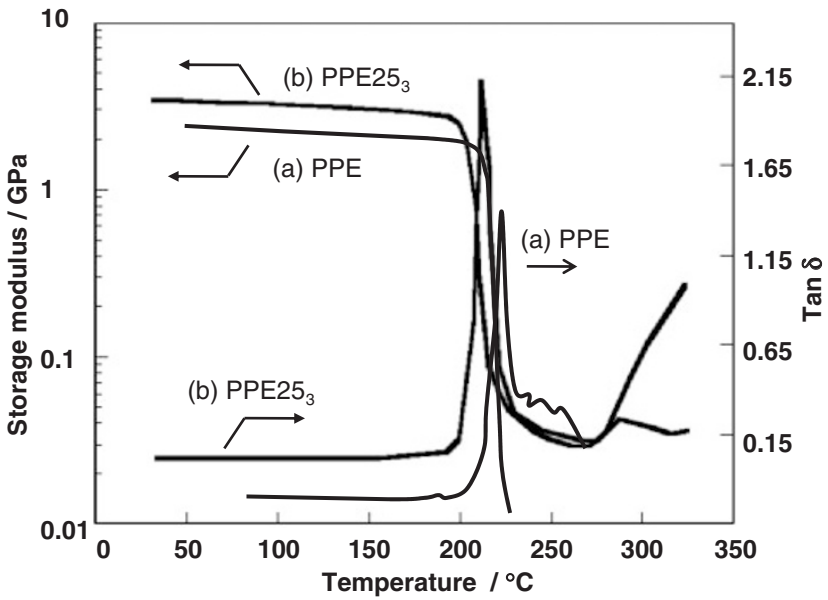

Figure 9. DMA profiles of (a) sample 11 (PPE) and (b) sample 8 (PPE25 ${ }_{3}$ ) $\left(2^{\circ} \mathrm{C} /\right.$ min under $\left.\mathrm{N}_{2}\right)$.

transparent films were obtained from copolymers ranging from PPE25 1 to PPE25 . This result also supports the higher thermostability of the copolymer.

Figure 9 shows the DMA profile of (a) PPE (sample 11) and (b) PPE25 3 (sample 8). From the maximum of the $\tan \delta\left(E^{\prime \prime} / E^{\prime}\right)$ curve, the glass transition temperatures can be estimated at 222 and $212^{\circ} \mathrm{C}$, respectively, which is in good agreement with the values determined by DSC measurement. It is noticeable that the DMA profile of PPE drops abruptly after the glass transition temperature, but that of PPE25 3 recovers at $250{ }^{\circ} \mathrm{C}$. After this temperature, the storage modulus $E^{\prime}$ of $\mathrm{PPE} 25_{3}$
Table V. Refractive index and dielectric properties of polymer

\begin{tabular}{rccccc}
\hline Sample & Abbreviation & $n^{\mathrm{a}}$ & $\begin{array}{c}\boldsymbol{\varepsilon} \\
(1 \mathrm{MHz})^{\mathrm{b}}\end{array}$ & $\begin{array}{c}\boldsymbol{\varepsilon} \\
(10 \mathrm{GHz})^{\mathrm{c}}\end{array}$ & Tan $\delta^{\mathrm{c}}$ \\
\hline 7 & PPE25 $_{5}$ & 1.57 & 2.45 & 2.35 & 0.0066 \\
9 & PPE25 $_{2}$ & 1.57 & 2.45 & 2.33 & 0.0032 \\
10 & PPE25 $_{1}$ & 1.57 & 2.45 & 2.31 & 0.0043 \\
11 & PPE & 1.57 & 2.45 & 2.38 & 0.0022 \\
\hline
\end{tabular}

${ }^{\text {a }}$ Refractive index at $\mathrm{D}$-line by Abbe refractometer. ${ }^{\mathrm{b}}$ Estimated value at $1 \mathrm{MHz}\left(\varepsilon=n^{2}\right)$. ${ }^{\mathrm{c}}$ Measured by cavity resonance method at $10 \mathrm{GHz}$.

rapidly increases, which suggests a crosslinking reaction occurs between the polymer chains probably because of the existence of the 25DMP unit. Although to specify the crosslinking point after gelation is difficult, the methylene coupling after the abstraction of benzyl proton of PPE25 $5_{x}$ or the $\mathrm{C}-\mathrm{C} / \mathrm{C}-\mathrm{O}$ coupling at ortho position of $\mathrm{OH}$ moiety of the 25DMP unit could be the most possible reaction. ${ }^{17}$

\section{Refractive Index and Dielectric Properties}

PPE offers potential as a next generation insulating material because of its excellent dielectric properties. Thus, to determine the influence of copolymerization with 25DMP on the dielectric properties, the refractive index $(n)$, dielectric constant $(\varepsilon)$, and dissipation factor $(\tan \delta$ ) were measured (Table V). All sample show a similar refractive index at around 1.57, and thus the estimated dielectric constant at $1 \mathrm{MHz}\left(\varepsilon=n^{2}\right)$ was almost the same. However, the dielectric constant at $10 \mathrm{GHz}$ measured by the cavity resonance method was somewhat different among the polymers. That is, conventional PPE gave a dielectric constant of 2.38 as compared to a value of 2.31 for PPE25. The dielectric constant increases with the feed of 25DMP, which could be a result of the well-packed structure of the 25DMP repeating unit. The realization of improved thermal and mechanical properties of the copolymer while maintaining its low dielectric properties would expand the application of this material as a next generation low $k$ insulator.

\section{SUMMARY}

2,5-Dimethylphenol was successfully copolymerized with 2,6-dimethylphenol using a di- $\mu$-hydroxo-bis $\left[\left(N, N, N^{\prime}, N^{\prime}\right.\right.$-tetramethylethylenediamine)copper (II)]chloride catalyst in toluene under oxygen. The conversion of the two monomers was monitored by gas chromatography, which revealed that 25DMP was slightly less reactive than 26DMP. The resulting copolymers show a higher thermal stability of about $70^{\circ} \mathrm{C}$ along with improved mechanical properties. This can be attributable to the 2,5-dimethylphenol unit, which is more likely to be located at the end of the copolymer.

Acknowledgment. This work was partially supported by the Tokuyama Science Foundation.

Received: July 22, 2009 Accepted: September 4, 2009 Published: December 15, 2009 


\section{REFERENCES}

1. A. S. Hay, Prog. Polym. Sci., 24, 45 (1999).

2. a) A. Rivaton and P. Morel, Polym. Degrad. Stab., 35, 3 (1992). b) A. Rivaton and P. Morel, Polym. Degrad. Stab., 35, 131 (1992). c) R. Frauke, S. Siegfried, and S. Ralf, Polym. Degrad. Stab., 65, 315 (1999).

3. N. S. Allen, Polym. Photochem., 1, 243 (1981).

4. B. T. Anthony and D. R. Olson, U. S. patent 4636408.

5. a) Y. Ishii and A. J. Ryan, Macromolecules, 33, 167 (2000). b) G. D. Cheney, M. E. Van Dyke, and S. J. Clarson, J. Inorg. Organomet. Polym., 8, 119 (1998).

c) J. Nunoshige, H. Akahoshi, Y. Liao, S. Horiuchi, Y. Shibasaki, and M. Ueda, Polym. J. 39, 828 (2007).

6. A. S. Hay, J. Polym. Sci., Part A: Polym. Chem., 36, 505 (1998).

7. A. S. Hay and G. F. Endres, Polym. Lett., 3, 887 (1965).

8. H. Higashimura, K. Fujisawa, Y. Moro-oka, S. Namekawa, M. Kubota, A. Shiga, H. Uyama, and S. Kobayashi, Macromol. Rapid. Commun., 21, 1121 (2000).
9. H. Higashimura, K. Fujisawa, Y. Moro-oka, M. Kubota, A. Shiga, H. Uyama, and S. Kobayashi, J. Mol. Catal. A: Chem., 155, 201 (2000).

10. H. Higashimura, K. Fujisawa, Y. Moro-oka, M. Kubota, A. Shiga, H. Uyama, and S. Kobayashi, Appl. Catal., A 194, 427 (2000).

11. H. Higashimura, K. Fujisawa, Y. Moro-oka, M. Kubota, A. Shiga, A. Terahara, H. Uyama, and S. Kobayashi, J. Am. Chem. Soc., 120, 8529 (1998).

12. Y. Shibasaki, M. Nakamura, R. Ishimaru, J. N. Kondo, and M. Ueda, Chem. Lett., 345, 662 (2005).

13. Y. Shibasaki, M. Nakamura, R. Ishimaru, J. N. Kondo, K. Domen, and M. Ueda, Macromolecules, 37, 9657 (2004).

14. Y. Shibasaki, M. Nakamura, J. N. Kondo, and M. Ueda, Macromol. Symp., 245/246, 87 (2006).

15. Y. Shibasaki, Y. Suzuki, and M. Ueda, Macromolecules, 40, 5322 (2007).

16. Y. Suzuki, Y. Shibasaki, and M. Ueda, Chem. Lett., 36, 1234 (2007).

17. A. Davis, Makromol. Chem., 132, 23 (1970).

18. J. E. Pickett, Polym. Degrad. Stab., 44, 119 (1994). 